\title{
Tratamientos biológicos anaerobios de residuos orgánicos como alternativa para la sostenibilidad energética en zonas Andinas - Ecuador
}

\section{Anaerobic biological treatments of organic waste as an alternative for energy sustainability in Andean areas - Ecuador}

\author{
Carlos Marcelo Matovelle Bustos ${ }^{1 *}$ y Moises Marcelo Matovelle Romo ${ }^{2}$ \\ ${ }^{1}$ Universidad Católica de Cuenca \\ 2 Universidad Católica de Cuenca, Sede Azogues. \\ *cmmatovelleb@ucacue.edu.ec
}

DOI: https://doi.org/10.26871/killkana_social.v2i3.328

\begin{abstract}
Resumen
La sostenibilidad energética es una interrogante que llama la atención a nivel mundial y cada vez es un blanco de investigación prioritaria. Empresas públicas, privadas, inversionistas y organizaciones sin fines de lucro ha venido debatiendo las mejores opciones de generación de energía, con alta eficiencia, bajo coste e impactos ambientales reducidos. Dentro de toda esta red de análisis, la generación de energía eléctrica a través del uso del biogás - metano como combustible producto de una digestión anaerobia de residuos sólidos orgánicos resulta ser una alternativa interesante para la sostenibilidad energética en zonas andinas. En esta investigación se presentan las perspectivas, interrogantes y retos que plantea esta alternativa energética desde un punto de vista económico y ambiental, para ello se utilizan resultados de simulaciones de generación eléctrica a partir de procesos de digestión anaerobia controlada, además de un análisis de esta tecnología como herramienta para el desarrollo sustentable en las zonas de estudio. Los resultados preliminares arrojan la eficiencia de esta tecnología con un costo elevado de inversión inicial que puede ser recuperado en el tiempo brindado opciones de manejo de residuos orgánicos, eficiencia energética, ingresos por venta de energía, impactos ambientales positivos y desarrollo en base de un ciclo cerrado en el cual se pueda hablar de sostenibilidad.
\end{abstract}

Palabras clave: generación eléctrica, digestión anaerobia controlada, sostenibilidad energética, zonas andinas.

\begin{abstract}
Energy sustainability is a question that draws attention worldwide and is increasingly a target of priority research. Public and private companies, investors and non-profit organizations have concluded that the best options for generating energy, with high efficiency, low cost and reduced environmental impacts. Within this network of analysis, the generation of electrical energy through the use of biogas - methane as a fuel product of an anaerobic digestion of organic waste is an interesting alternative for energy sustainability in Andean areas. This research presents the perspectives, questions and challenges posed by this energy alternative from an economic and environmental point of view, for which the results of the simulations of electric generation are used from controlled anaerobic digestion processes. of an analysis of this technology as a tool for sustainable development in the study areas. The preliminary results show the efficiency of this technology with a high investment cost that can be recovered over time, provide solutions for organic waste management, energy efficiency, income from energy sales, positive environmental impacts and development based on a closed cycle in which you can talk about sustainability.
\end{abstract}

Key words: electrical generation, controlled anaerobic digestion, energy sustainability, Andean areas.

\section{Introducción}

Se define a la sostenibilidad energética como el equilibrio entre tres dimensiones principales: la seguridad energética, la sostenibilidad ambiental y la equidad social (World Energy Monitor Issues, 2015). En otras palabras, se trata de llevar el concepto de equilibrio entre las di- mensiones económica, social y ambiental, propuesto por desarrollo sostenible, al plano energético, reconociendo que la energía es clave y básica para el desarrollo de cualquier sociedad (Rojas Wang, 2007). En la Quinta Cumbre de las Américas se reconoció que la energía es un recurso esencial para mejorar el nivel de vida de nuestros pueblos, y que 
el acceso a la energía es de primordial importancia para el crecimiento económico con equidad e inclusión social (Organización de los Estados Americanos, 2009).

La seguridad energética aborda una gestión efectiva y fiable de los recursos energéticos domésticos y externos; la equidad social busca que la energía sea accesible y asequible para toda la población; y la sostenibilidad ambiental requiere la mitigación de los impactos negativos por medio del desarrollo de fuentes de energía limpias y bajas en emisiones de carbono (Rojas Wang, 2007).

El Documento Final de la Conferencia de las Naciones Unidas sobre Desarrollo Sostenible contiene la Declaración de Río+20 "El Futuro que Queremos“ (EEAS, 2018), en el cual se considera a la energía como una de las esferas temáticas incluida simultáneamente en las cuestiones intersectoriales y en el marco para la acción y seguimiento de las estrategias de desarrollo sostenible (Instituto Interamericano de Cooperación para la Agricultura (IICA), 2015).

El crecimiento económico y social del Ecuador, el avance y desarrollo de la industria, la tecnología y la evolución de los estilos de vida de la población hacen indispensable la planificación estratégica permanente del sector energético. Los hidrocarburos, la electricidad y la energía renovable requieren ser tratadas íntegramente bajo una política que estimule el uso eficiente de los recursos y el ahorro. Todo, con el objetivo de garantizar el abastecimiento de energía en el corto, mediano y largo plazo que permita satisfacer la demanda de las presentes y futuras generaciones (Rosero y Chiliquinga, 2011)

Una gran zona de interés dentro del análisis de la sostenibilidad energética es las zonas andinas rurales de Ecuador. Estos lugares por características particulares presentan problemas energéticos que no han sido atendidos en su totalidad, ya sea por su el difícil acceso a la interconexión de las redes de distribución energética, la topografía de la zona, la dispersión de las comunidades dentro del espacio e inclusive las condiciones climáticas.

Las zonas andinas ecuatorianas se caracterizan por su producción ganadera por lo que adicionalmente tienen de cara un problema ambiental con la generación de residuos sólidos orgánicos como desecho producido por el estiércol de animales de granja.

La producción de energía eléctrica a partir de biomasa se convierte en una atractiva estrategia para el manejo de la demanda, ya que no solo permitiría suplir en parte las necesidades del consumo de energía eléctrica sino que también disminuiría la demanda nacional. Asimismo, permitiría el tratamiento y manejo de los desechos orgánicos en fincas agropecuarias, contribuyendo al cumplimiento de las obligaciones ambientales (Acosta y Obaya Abreu, 2005).

\subsection{Perspectivas de la comunidad andina y de las zonas rurales}

El contexto energético andino se caracteriza por la abundancia de recursos, en especial hidrocarburos y car- bón, que representan una importante fuente de ingresos a la región. En las exportaciones de los países andinos al mundo, en el sector energético representa un $52 \%$ del total de las exportaciones comunitarias. Las reservas probadas de petróleo en la comunidad andinas son de aproximadamente 85 billones de barriles y para el gas natural las reservas probadas alcanzan más de 5 mil millones de metros cúbicos. Se estima que pueden existir todavía un volumen importante de reservas todavía por descubrir. El carbón mineral es un recurso que se encuentra principalmente en Colombia y Venezuela con cerca de 8 mil millones de toneladas en reserva de alta calidad con un alcance estimado de 171 años. (Organización Latinoamaericana de Energía, 2003)

De una manera general, contando con una variedad de contextos y recursos energéticos, Ecuador no ha desarrollado fuertemente la utilización de energías renovables. Sin embargo, existe un fuerte interés de incrementar la presencia de estas fuentes en la matriz energética ecuatoriana. (Horta, 2005)

La utilización de energías renovables en el Ecuador ha sido liderada por el Instituto Nacional de Eficiencia Energética y Energías Renovables (INER), que es un Instituto Público de Investigación, encargado de la generación de conocimiento científico tecnológico y el fortalecimiento de capacidades locales en el ámbito de la eficiencia energética y energías renovables. Contribuyendo a la toma de decisiones para la diversificación de la matriz energética, el cambio de la matriz productiva y la mitigación del cambio climático. (Instituto Nacional de Eficiencia Energética y Energías Renovables, 2015).

Diversos estudios se realizan en el Ecuador para determinar fuentes potenciales de energías renovables. En los estudios de los potenciales energéticos de la biomasa se evaluó que los residuos agrícolas podrían generar el $50 \%$ de la presente demanda de electricidad, mientras que los desechos municipales y residuos animales podrían generar respectivamente $16 \%$ y $42 \%$ de las necesidades de energía eléctrica. (Horta, 2005)(Sanchez, 2004)

Paradójicamente, estas regiones cuentan con diversos recursos naturales que pueden ser aprovechados para mejorar el cubrimiento del servicio de energía con incentivos al desarrollo de estrategias tecnológicas basadas en energías alternativas, tales como el uso de la biomasa y las energías hidráulica, eólica y solar, cuyo potencial no es conocido, salvo en casos puntuales de algunos proyectos de investigación. Entre las principales causas de los problemas energéticos en las regiones no interconectadas, se encuentra la falta de información técnica sobre las condiciones del sistema de distribución, la deficiente estimación de la demanda, la falencia en posibles soluciones alternativas a la conexión al sistema central, el costo elevado y la difícil sostenibilidad de las soluciones basadas en combustibles líquidos como el diésel. Por otra parte, la carencia de estudios y mediciones reales de los potenciales energéticos de estas zonas, dificulta el planteamiento de proyectos encaminados 
a suplir energía por métodos distintos a los convencionales. (Pantoja, Guerrrero, y Fajardo, 2015)

Dentro de estos datos presentados queda la incógnita de como satisfacer las demandas energéticas en las zonas andinas que no están conectadas a la red de distribución y de cómo utilizar las potencialidades que tienen estas zonas para de esta manera convertir sus residuos generados en energía que pueda ser utilizada y hasta en ciertos casos vendida.

\section{Metodología}

Para el desarrollo de la investigación se realiza simulaciones de digestión anaerobia y de producción de metano utilizando el programa DESSAS 7.1, que es un programa concebido para la depuración de aguas residuales pero que tiene integrados módulos que facilitan el planteamiento de varias alternativas. El esquema que se propone cuenta con un decantador primario, un espesador de fangos y un digestor anaerobio (Matovelle, Macancela, Icaza, y Quito-Abad, 2018), esto permite simular las condiciones de generación de residuos sólidos en las granjas de análisis.

La zona de estudio se ubica en la Parroquia Tarqui, perteneciente a la ciudad de Cuenca en el sur del Ecuador. Tarqui se caracteriza por se una zona ganadera en donde prima la producción lechera, con una temperatura promedio alrededor de los $14^{\circ} \mathrm{C}$, resulta ser la zona ideal para realizar la investigación. Se han analizado la producción de residuos de 510 animales de granja divididos entre vacas de producción lechera, puercos, ovejas y caballos. Existen datos de la cantidad de residuos sólidos generados en 4 haciendas de estudio que se encuentran cercanas y que se podría analizar las ventajas de la producción de biogás y de tratamiento de sus residuos sólidos generados.

Existen datos de la cantidad de residuos sólidos que genera de acuerdo al tipo de ganado y la cantidad de gas que puede producir esos residuos como se puede ver en la Tabla 1 (Achinas, Achinas, y Euverink, 2017). Para el análisis $\mathrm{y}$ simulaciones que plantea este trabajo solamente se ha considerado la cantidad de residuos sólidos y con los datos de materia orgánica producida se realiza la simulación de producción de metano.

Tabla 1 Potencial de producción por biogás por animal

\begin{tabular}{|c|c|c|c|}
\hline $\begin{array}{c}\text { Tipo de } \\
\text { ganado }\end{array}$ & $\begin{array}{c}\text { Unidad de } \\
\text { animal } \\
\text { equivalente } \\
\text { UAE }\end{array}$ & $\begin{array}{c}\text { Cantidad de } \\
\text { residuos } \\
\text { sólidos }\left(\mathrm{m}^{3} /\right. \\
\text { animal })\end{array}$ & $\begin{array}{c}\text { Producción de } \\
\text { gas } \\
\left(\mathrm{m}^{3} / \text { UAE/día }\right)\end{array}$ \\
\hline $\begin{array}{c}\text { Vacas de } \\
\text { producción } \\
\text { lechera }\end{array}$ & 1.2 & 0.055 & $0.56-1.5$ \\
\hline Porcinos & 0.12 & 0.045 & $0.6-1.25$ \\
\hline Ovinos & 0.1 & 0.003 & \\
\hline Equinos & 1.1 & 0.023 & \\
\hline
\end{tabular}

La digestión anaerobia controlada es un proceso en el cual se puede mantener la temperatura ideal para lograr la mayor eficiencia, al momento de plantear simulaciones previas al desarrollo de un prototipo se puede ahorrar tiempo y recursos porque se trabaja con un rango de resultados óptimos. Luego de verificar los tiempos de retención y la temperatura adecuada de digestión se analiza la producción de metano y la generación eléctrica, el primer uso que se le da a la electricidad producida es para lograr calentar los digestores y luego para la iluminación de las viviendas que se encuentran dentro de los predios de las haciendas analizadas (Matovelle y cols., 2018).

Se utiliza el programa DESSAS 7.1, para realizar varias simulaciones de digestión y generación de acuerdo a los criterios de diseño para digestión anaerobia, se utiliza los criterios de diseño de temperatura, tiempo de retención celular y crecimiento bacteriológico ya que el programa permite la introducción y modificación de estos parámetros además de características constructivas como materiales y dimensiones (Matovelle y cols., 2018).

La producción de biogás mediante el uso de simulaciones ha sido analizada por (Matovelle y cols., 2018), con esos datos se plantea el análisis energético en las zonas de estudio en donde se realizaron las simulaciones de generación eléctrica a partir de procesos de digestión anaerobia controlada.

Se ha analizado los consumos que tiene el proceso de digestión, identificando como los principales al consumo por la agitación y por la calefacción necesaria para llevar al proceso a los $35^{\circ} \mathrm{C}$. También se han calculado la eficiencia de los motores de combustión interna que se utilizan para la generación de energía eléctrica a través del biogás con potencia eléctrica de $335 \mathrm{~kW}$ es de $36.3 \%$ (GE Power Generation, 2017), aunque la eficiencia disminuyó en las simulaciones por las condiciones ambientales de la zona. No se realizan simulaciones del generador eléctrico (datos teóricos) se considera un valor de eficiencia del transformador de $30 \%$ como un dato conservar para no mostrar resultados muy elevados y que en la realidad no pudieran alcanzarse (Vera-Ronero, Martínez-Reyes, Estrada-Jaramillo, y OrtizSoriano, 2014).

De acuerdo con datos de fabricantes de motores para agitación (AQUALIMPIA, 2013), se establece que la potencia del motor debe ser de $1 \mathrm{~kW}$ por cada $100 \mathrm{~m} 3$. En la Tabla 2 se muestran las especificaciones técnicas del motor.

Tabla 2 Especificaciones de los motores de agitación

\begin{tabular}{|c|c|c|c|c|}
\hline $\begin{array}{c}\text { Potencia } \\
\text { de salida } \\
\mathrm{kW}\end{array}$ & $\begin{array}{c}\text { Frecuencia } \\
\mathrm{Hz}\end{array}$ & $\begin{array}{c}\text { Modelo } \\
\text { de motor }\end{array}$ & Eficiencia & $\mathrm{kWh}$ \\
\hline 0.28 & 60 & $2256 \mathrm{M} 6$ & 0.9 & 6.72 \\
\hline
\end{tabular}

Se ha considerado el escenario más desfavorable de consumo, que es en el cual el agitador funcione las 24 horas del día, obteniendo un consumo de $6.72 \mathrm{kWh}$ en el día de funcionamiento.

El segundo consumo energético de importancia dentro del proceso es el que realiza el motor térmico para llevar 
al biodigestor a la temperatura de $35^{\circ} \mathrm{C}$ para realizar el proceso de digestión anaerobia. Para realizar el cálculo se realizan las consideraciones que se presentan en la Tabla 3, en donde se analiza las características del material, en las simulaciones se realizó los cálculos para fibra de vidrio con un espesor de $0.01 \mathrm{~m}$ y se considera la temperatura más desfavorable en la que tenga que empezar el Biodigestor hasta llegar a los $35^{\circ} \mathrm{C}$.

Tabla 3 Datos biodigestor

\begin{tabular}{|l|c|}
\hline Material & Fibra de vidrio S-2 \\
\hline Volumen $(\mathbf{m 3})$ & 0.082 \\
\hline Densidad $(\mathrm{g} / \mathbf{c m 3})$ & 2.46 \\
\hline Calor específico $\left(\mathbf{J} / \mathbf{k g}{ }^{\circ} \mathbf{K}\right)$ & 795 \\
\hline Temperatura inicial $\left({ }^{\circ} \mathbf{C}\right)$ & 5 \\
\hline Temperatura inicial $\left({ }^{\circ} \mathbf{C}\right)$ & 35 \\
\hline
\end{tabular}

\section{Resultados y Discusión}

Se ha calculado la producción de residuos sólidos que generan los animales de la zona de estudio, de esta manera se tiene un volumen con el cual se puede trabajar según los tiempos de retención plateados en la simulación, la producción de residuos para las haciendas de análisis es de $21.06 \mathrm{~m}^{3}$, lo que indica que es necesario es un volumen mínimo del tanque digestor para lograr un proceso adecuado, sin olvidar considerar que ese el volumen que se genera en un día y el tiempo de retención celular es 20 días. Los tiempos de retención elevados generarían un volumen mayor de construcción por lo que (Matovelle y cols., 2018) proponen un sistema con recirculación del fango que se está tratando, obteniendo varios escenarios que han sido simulados. Se ha trabajado con el valor de una de las simulaciones presentadas en el estudio mencionado en la que 1 momento de aumentar la carga de concentración de materia orgánica la reacción de digestión anaerobia produce mayor cantidad de metano, esta simulación da como resultado un volumen de alrededor de $18.5 \mathrm{~m} 3$ en un día, con los mismos volúmenes de reactor y con el uso de la misma energía para llegar a la temperatura de trabajo de $35^{\circ} \mathrm{C}$. En la Figura 1 se observa los datos de producción de metano

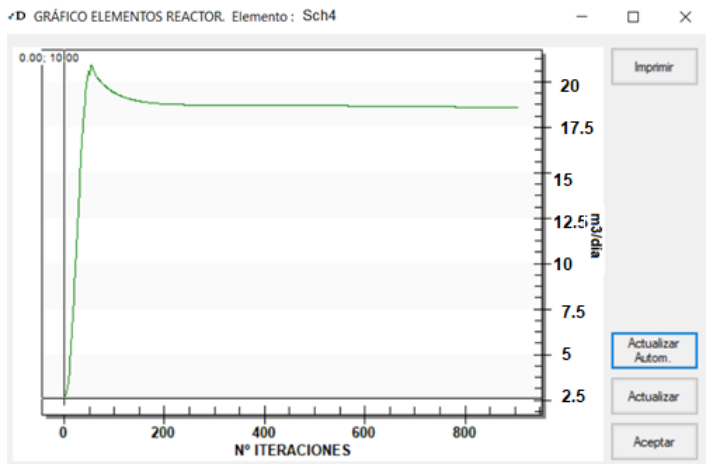

Figura 1. Simulación de producción de metano
Al obtener una generación de $18.5 \mathrm{~m}^{3}$ /día de $\mathrm{CH}_{4}$ con una energía de biogás de 28.947 MWh (Vera-Ronero y cols., 2014) y producción eléctrica de $120.3 \mathrm{kWh} /$ día con $255 \mathrm{~kg}$ de DBO/día (Matovelle y cols., 2018) medidos en la cantidad de residuos sólidos generados. Se debe tomar en cuenta que el primer año del proyecto aún no genera energía eléctrica, ya que está asignado a la parte de la construcción y puesta en marcha.

En la Figura 2 se muestra el esquema y especificaciones básicas del biodigestor propuesto.

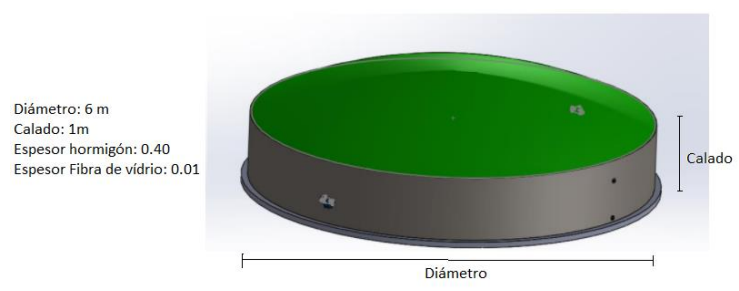

Figura 2. Biodigestor propuesto en el análisis

El cálculo para el consumo energético se presenta a continuación (Ecuación 1):

$$
Q=m * C_{A} * \Delta T
$$

$$
Q=4811022 J=1,35 k w h
$$

Para determinar el total de las inversiones para el presente proyecto se han considerado los costos de las respectivas adecuaciones y construcciones de las maquinarias y equipos tales como son los biodigestores, tanques y lechado de secado de lodo. A continuación, se indican las dimensiones de cada una de las partes a invertir, ya que basado en ello se establecen los costos.

Tabla 4 Elementos dentro de la propuesta

\begin{tabular}{cccccc}
\hline Descripción & $\begin{array}{c}\text { Diámetro } \\
(\mathrm{m})\end{array}$ & $\begin{array}{c}\text { Altura } \\
(\mathrm{m})\end{array}$ & $\begin{array}{c}\text { Largo } \\
(\mathrm{m})\end{array}$ & $\begin{array}{c}\text { Ancho } \\
(\mathrm{m})\end{array}$ & $\begin{array}{c}\text { Volumen } \\
\left(\mathrm{m}^{3}\right)\end{array}$ \\
\hline $\begin{array}{c}\text { Espesador } \\
\text { inicial }\end{array}$ & 3 & 0.75 & & & 5.3 \\
\hline Biodigestor & 6 & 1 & & & 28.77 \\
\hline $\begin{array}{c}\text { Tanque de } \\
\text { descarga }\end{array}$ & 0.5 & 2 & 2 & 8 \\
\hline $\begin{array}{c}\text { Lecho de } \\
\text { secado de } \\
\text { lodos }\end{array}$ & 0.5 & 5 & 5 & 12.5 \\
\hline
\end{tabular}

En el presente estudio se omiten los detalles del presupuesto de obra y solamente se colocan los datos de los valores finales, mostrados en la Tabla 5. 
Tabla 5 Inversión requerida para la construcción del sistema del biodigestor para generación de energía eléctrica.

\begin{tabular}{lccc}
\hline \multicolumn{1}{c}{ Descripción } & Cantidad & $\begin{array}{c}\text { Precio } \\
\text { unitario } \\
\text { USD }\end{array}$ & $\begin{array}{c}\text { Precio } \\
\text { total }\end{array}$ \\
\hline USD & 1 & 5200 & 5200 \\
\hline Espesador inicial & 1 & 60594 & 60594 \\
\hline Biodigestor & 1 & 3100 & 3100 \\
\hline Tanque de descarga & 1 & 3924 & 3924 \\
\hline Lecho de secado de lodos & 1 & 1000 & 1000 \\
\hline Quemador de biogás & 1 & 5000 & 5000 \\
\hline Filtros & 1 & 5000 & 5000 \\
\hline Generador eléctrico & 1 & 3000 & 3000 \\
\hline $\begin{array}{l}\text { Exteriores (jardinería, ac- } \\
\text { ceso, seguridad) }\end{array}$ & & & $\mathbf{8 6 8 1 8}$ \\
\hline TOTAL & & & \\
\hline
\end{tabular}

Los ingresos económicos por la energía generada se muestran en la Tabla 6, en donde se restan los gastos por el consumo que existe en cada uno de los elementos, considerando ya la eficiencia y las condiciones climáticas.

Tabla 6 Ingresos por la energía generada.

\begin{tabular}{lc}
\hline Generación kWh/día & 120.3 \\
\hline Consumo agitación kWh/día & 6.72 \\
\hline Consumo calefacción $\mathrm{kWh} /$ día & 1.34 \\
\hline Pérdidas en generación kWh/día & 18.5 \\
\hline Consumos de las granjas kWh/día & 50 \\
\hline Energía disponible & 43.74 \\
\hline Ingresos diarios USD & $\mathbf{4 . 2}$ \\
\hline
\end{tabular}

Los datos de la Tabla 4, señalan una posible sostenibilidad energética dentro de la zona de análisis pues se satisfacen todas las demandas de energías de las granjas de la zona, con la generación eléctrica producto de la combustión del biogás obtenido en la degradación anaerobias de sus residuos sólidos orgánicos.

\section{Conclusiones}

La degradación de residuos sólidos orgánicos proveniente de animales tiene la capacidad de generar biogás metano si se la realiza en condiciones de temperatura y presión contralados para que se viable en la zona de análisis en la cual la temperatura ambiente podría producir procesos de degradación muy lentos y en los cuales las bacterias no logren tazas de rendimientos aprovechables. Además de ser una solución al tratamiento de residuos sólidos generados por las actividades de ganadería, resulta ser una solución a los problemas energéticos que sufre la zona de estudio.

Las simulaciones de producción de metano arrojan cantidades aprovechables de gas que puede ser utilizado en la generación eléctrica, las necesidades energéticas para lograr este proceso controlado y con eficiencias aceptables son elevadas provocando que la cantidad de energía disponible disminuya, pero que luego de satisfacer las necesidades energéticas primordiales de las zona de estudio, quede un remanente energético que pueda ser conectado a la red pública y que inclusive pueda ser comercializado representando ingresos para las zonas.

Los ingresos diarios representan una cantidad tan pequeña que se necesitaría de alrededor de 58 años para recuperar la inversión de la construcción y operación del biodigestor, económicamente resulta una mala inversión, pero para hablar de Desarrollo Sostenible se debe dejar las matemáticas y números planos y pensar más a fondo en los verdaderos objetivos que se busca con la investigación y desarrollo, estos son solucionar problemas comunes de gente común y con esta propuesta de energía renovable aplicada en zonas en las que la demanda energética pueda ser no satisfecha se está logrando tratar el problema ambiental de generación de residuos sólidos, satisfacer las necesidades energéticas y además crear un ingreso adicional que puede ser invertido en la mejora y desarrollo tecnológico.

\section{Referencias Bibliográficas}

Achinas, S., Achinas, V., y Euverink, G. J. W. (2017). A Technological Overview of Biogas Production from Biowaste. Engineering, 3(3), 299-307. doi: 10.1016/ J.ENG.2017.03.002

Acosta, Y. L., y Obaya Abreu, M. C. (2005). La digestión anaerobia. Aspectos teóricos. Parte 1. Instituto $\mathrm{Cu}$ bano de Investigaciones de los Derivados de la Caña de Azúcar (ICIDCA), XXXIX(1), 35-48.

AQUALIMPIA. (2013). PLANTAS DE BIOGÁS - BIODIGESTORES INDUSTRIALES.

EEAS. (2018). EU-CELAC : A Strategic Partnership for the 21 st century.

GE Power Generation. (2017). Jenbacher type 2 fact sheet (Vol. 2001; Inf. Téc.).

Horta, L. (2005). Perspectivas de sostenibilidad energética en los países de la Comunidad Andina (Vol. 83) (n. ${ }^{\circ}$ 3). doi: 10.3989/arbor.2000.i653.1000

Instituto Interamericano de Cooperación para la Agricultura (IICA). (2015). Uso y acceso a las energías renovables en territorios rurales (O. Vega, Ed.). San José: Imprenta del IICA.

Instituto Nacional de Eficiencia Energética y Energías Renovables. (2015). Dossier INER (Inf. Téc.).

Matovelle, C., Macancela, D., Icaza, D., y Quito-Abad, A. (2018). Simulación de generación eléctrica mediante un proceso de digestión anaerobia controlada de residuos sólidos orgánicos. INVESTIGAR, 10-15.

Organización de los Estados Americanos. (2009). Quinta Cumbre Puerto España 2009. , 14.

Organización Latinoamaericana de Energía. (2003). No Title.

Pantoja, A., Guerrrero, K., y Fajardo, D. (2015). Análisis de oportunidades energéticas con fuentes alternativas en el de Departamento de Nariño. , 38-51.

Rojas Wang, J. P. (2007). La sostenibilidad energética. Energía y regulación(289), 171-179. 
Rosero, E., y Chiliquinga, B. (2011). Energías Renovables Observatorio de Energías Renovables en América Latina y el Caribe., 1-92.

Sanchez, S. (2004). Las energías renovables en Ecuador. En Presentación power point.

Vera-Ronero, I., Martínez-Reyes, J., Estrada-Jaramillo, M., y Ortiz-Soriano, A. (2014). Potencial de generación de biogás y energía eléctrica Parte I: excretas de ganado bovino y porcino. Ingeniería, Investigación y Tecnología, 15(3), 429-436. doi: 10.1016/S1405 -7743(14)70352-X

World Energy Monitor Issues. (2015). World Energy Issues Monitor. World Energy Council, 1-40. 